\title{
Ten Commandments of Safe and Optimum Mastectomy for Breast Cancer
}

\author{
Chintamani
}

Published online: 19 July 2014

(C) Association of Surgeons of India 2014

There has been a paradigm shift in the management of breast cancer in the last century and especially in the last two decades. Contrary to the Halsteadean concept of an aggressive local approach, it is now generally believed that "less is more" based on the understanding that breast cancer is predominantly a systemic disease. Although most centers across the globe practice breast conservation surgery for early breast cancer, the same may not be true for the developing world where modified radical mastectomy (MRM) is still the most commonly performed surgery for this disease [1].

This is primarily due to the fact that majority of breast cancers in the developing countries are locally advanced at presentation. These cancers may actually require some form of neoadjuvant chemotherapy to make them resectable [2-4]. This operation (MRM), however, is still the workhorse, and most surgeons must be trained to perform an optimum and oncologically safe MRM. Like there are many ways to skin a cat, there indeed are various "correct" ways of performing this procedure and every surgeon has to be familiar with at least one such way of doing it. These "commandments" are by no means the only vital steps during this operation but are important and key guiding points (Table 1).

Chintamani $(\bowtie)$

Vardhman Mahavir Medical College, Safdarjang Hospital, New Delhi, India

e-mail: chintamani7@rediffmail.com
Table 1 Ten commandments of MRM

Thou shall...

1. Drape and position the patient correctly

2. Raise the flaps of optimum thickness

3. Shall dissect in a medial to lateral direction and reach the lateral border of pectoralis major

4. Shall find medial and lateral pectoral nerves and preserve them

5. Shall preserve and lift the pectoralis minor on a sling

6. Shall dissect all the levels of axilla (level-I-III)

7. Shall attempt to preserve all intercostobrachial nerves

8. Shall reach the medial (Halstead's Ligament) lateral (thoracodorsal pedicle) and lower limits (angular vein) of axillary lymph node dissection.

9. Shall achieve and demonstrate the end points of axillary dissection to the surgical assistants

10. Shall close the wound and leave half vacuum suction drains in situ

\section{Commandment-1—Positioning Appropriately}

The patient is usually kept supine with a thin sandbag under the ipsilateral scapula to facilitate axillary lymph node dissection (ALND). The sandbag pushes the posterior wall and floor of axilla anteriorly. It is advisable to drape the ipsilateral upper limb separately in order to keep it free for adduction of arm that may be required during ALND to relax and retract the pectoralis major for dissection of level-III lymph nodes (Fig. 1). If some reconstructive procedure like latissimus dorsi flap (LD flap) is also planned along with mastectomy, the position of the patient may require to be changed during surgery into a semi-prone or lateral position. Some surgeons (including the author) like to perform the entire procedure in a lateral position if the LD flap is contemplated. 


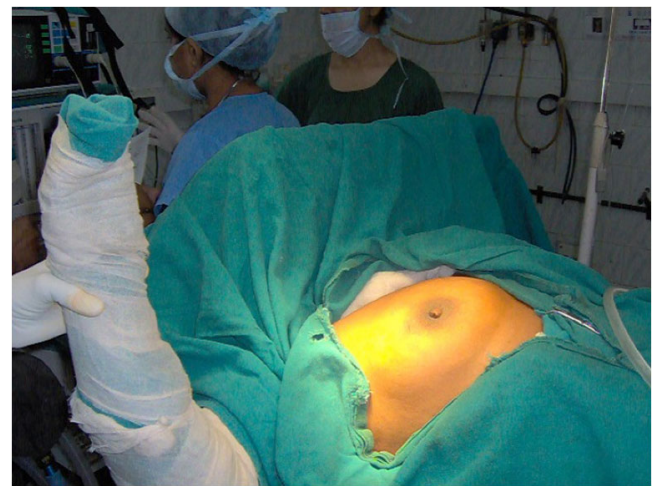

Fig. 1 Positioning: The patient is kept in a supine position with a thin sandbag under the ipsilateral scapula to facilitate axillary dissection. The ipsilateral arm is draped separately and kept free for adduction during axillary dissection

\section{Commandment-2-Making the Right Incision}

Classically a transverse incision (incorporating the nipple areola complex and an ellipse of skin with a margin of at least $2 \mathrm{~cm}$ from the palpable tumor) is made. The incision extends from lateral sternal edge to the anterior axillary line (Fig. 2). It is recommended not to extend the incision beyond the anterior axillary line in order to prevent inadvertent irradiation of axilla during adjuvant radiotherapy (the operative scar may have to be included in the field). There are various modifications of this incision that are used and may also vary depending upon the location of the tumor. There is a general consensus now that it is not mandatory to remove the grossly uninvolved skin. There is a type of mastectomy where the skin envelope (with or without nipple areola complex) overlying the breast may be preserved (skin sparing mastectomy). This may be followed by immediate breast reconstruction with or without an implant.

\section{Commandment-3—Raising the Flaps Optimally}

Most surgeons prefer to raise the superior flap first, but it would depend on the operator's choice and no significant difference has been observed in the outcome. The aim is to get the right thickness of skin flaps, i.e., neither too thin nor thick. The superior flap is raised up to the clavicle while the

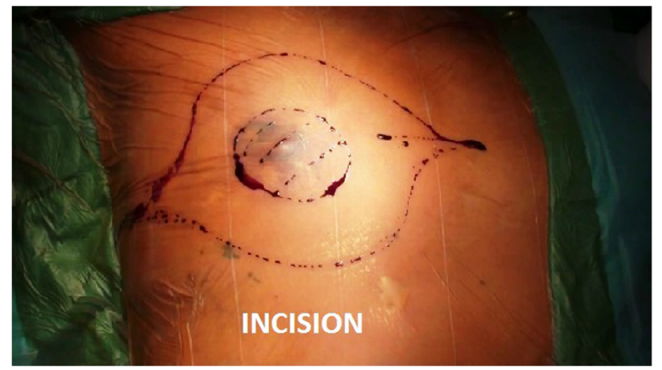

Fig. 2 Making an incision: an elliptical incision including the overlying skin with a margin of $2 \mathrm{~cm}$ from the palpable edge of the tumor lower one is lifted right up to the inframammary fold. The effort should aim at accomplishing a near impossible task of leaving no breast tissue on the flaps. This is feasible if one adheres to a plane between the smaller fat lobules of subcutaneous tissue and the larger breast fat lobules. This, however, becomes difficult in obese patients where the subcutaneous fat lobules may be larger than usual. The author relies on a relatively easier method of achieving this by ensuring that the sub-dermal plexus of vessels remains on the flap (Fig. 3). Having used this technique, the author has observed a very low incidence of flap necrosis in his study [3]. It is convenient to perform the medial dissection first as there is minimal breast tissue present there and one may reach the pectoral fascia easily. The medial perforators are looked for to be ligated or diathermized. The dissection now proceeds laterally and the weight of the breast provides the much needed traction to facilitate dissection in a relatively blood less plane between pectoral fascia and the pectoralis major muscle (PM). Some deep perforators from intercostal vessels may be encountered but they can be easily controlled. Raising the lower flap of optimum thickness is technically more challenging, as the breast tissue is closer to skin here and needs extra care to prevent buckling or a thick flap. Skin hooks or Allis tissue holding forceps are handy for applying traction to facilitate the raising of flaps (Fig. 3). The breast along with intact pectoral fascia is now dissected off the PM from a medial to lateral direction up to the lateral border of PM (Fig. 4a, b). The lateral border of PM is dissected in an upward direction, and the pectoralis minor muscle comes into view as soon as the PM is retracted using a Morris retractor. The pectoralis minor is dissected right up to its insertion at coracoid process. This maneuver also exposes the interpectoral space (that houses the inter-pectoral group or Rotter's nodes that are also included in the level-II group). The fibro-fatty tissue along with nodes in this region needs to be dissected and removed. The axillary dissection thus involves clearing off the superior and inferior surfaces of pectoralis minor. This step facilitates the next two very important aspects of axillary dissection. The pectoral

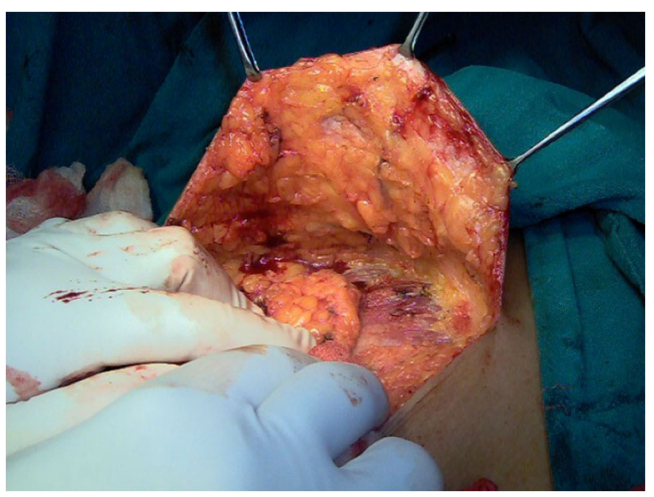

Fig. 3 Raising the flaps using traction and counter-traction. The dermal plexus can be seen. This plexus ensures that the flap is optimum thickness and also does not necrose 

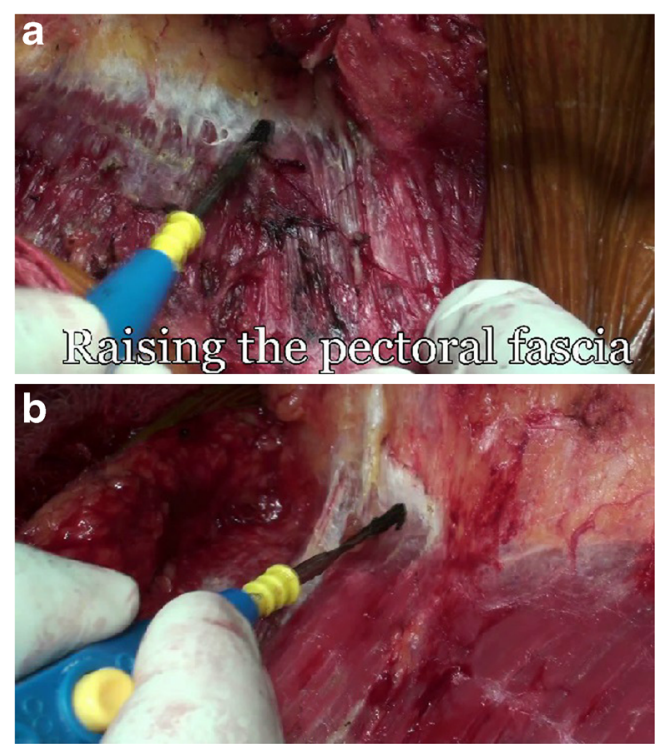

Fig. 4 a The pectoral fascia being lifted off the PM in a medial to lateral direction. The plane is blood less if the traction is applied on the breast tissue. One may use diathermy or a cold knife or scissors. b The pectoral fascia being lifted off the PM in a medial to lateral direction. The Cooper's ligament may be seen

nerves can now be seen clearly and the pectorals minor muscle can now be dissected and lifted from the chest wall and taken on a sling to facilitate the level-II and III dissection (Fig. 5a).

\section{Commandment-4-Looking for Medial and Lateral Pectoral Nerves (Fig. 5)}

The medial and lateral pectoral nerves are now identified and preserved. The medial pectoral nerve is actually "lateral" in anatomical location and is so named based on the cord from where it descends. Preserving these nerves is vital if one is preserving the pectoralis major and minor muscles.

\section{Commandment-5-Preserving and Retracting the Pectoralis Minor Muscle}

The pectoralis minor muscle is routinely preserved along with PM these days (the Patey's MRM that involved cutting of the pectoralis minor muscle to facilitate level-III axillary dissection is no longer routinely practiced). During this step, the adduction of ipsilateral arm relaxes the PM and allows the retraction of pectoralis minor muscle laterally to facilitate level-III axillary dissection (Fig. 6).

\section{Commandment-6-Performing Complete Dissection of Axilla (Level-I to III)}

Although there is enough evidence to indicate that axillary dissection up to level-II is adequate in patients with node negative axillae (as the incidence of involvement of level-III nodes in the absence of level-I disease is less than $5 \%$ ). However, the axillary burden in the developing countries makes it desirable to dissect up to level-III. This also ensures an optimum level-II dissection. The author routinely performs ALND up to level-III.

Conventionally, the medial limit of level-III dissection is considered to be "Halstead's ligament" or the "Costo-clavicular ligament," while the lateral limit is the medial border of the non-retracted pectoralis minor muscle. The upper limit of this dissection is axillary vein and the use of diathermy close to the vein is not recommended. It is also desirable not to
Fig. 5 After reaching the lateral border of PM, the muscle is completely dissected and lifted on the Morris retractor. The medial and lateral pectoral nerves may be seen. The "medial nerve" is lateral in anatomical location as can be seen. a The pectoralis minor is lifted after dissection right up to the coracoid process and lifted on a sling. b The pectoral pedicle made by the pectoral branch of Thoracoacromian artery can be seen. It must be preserved
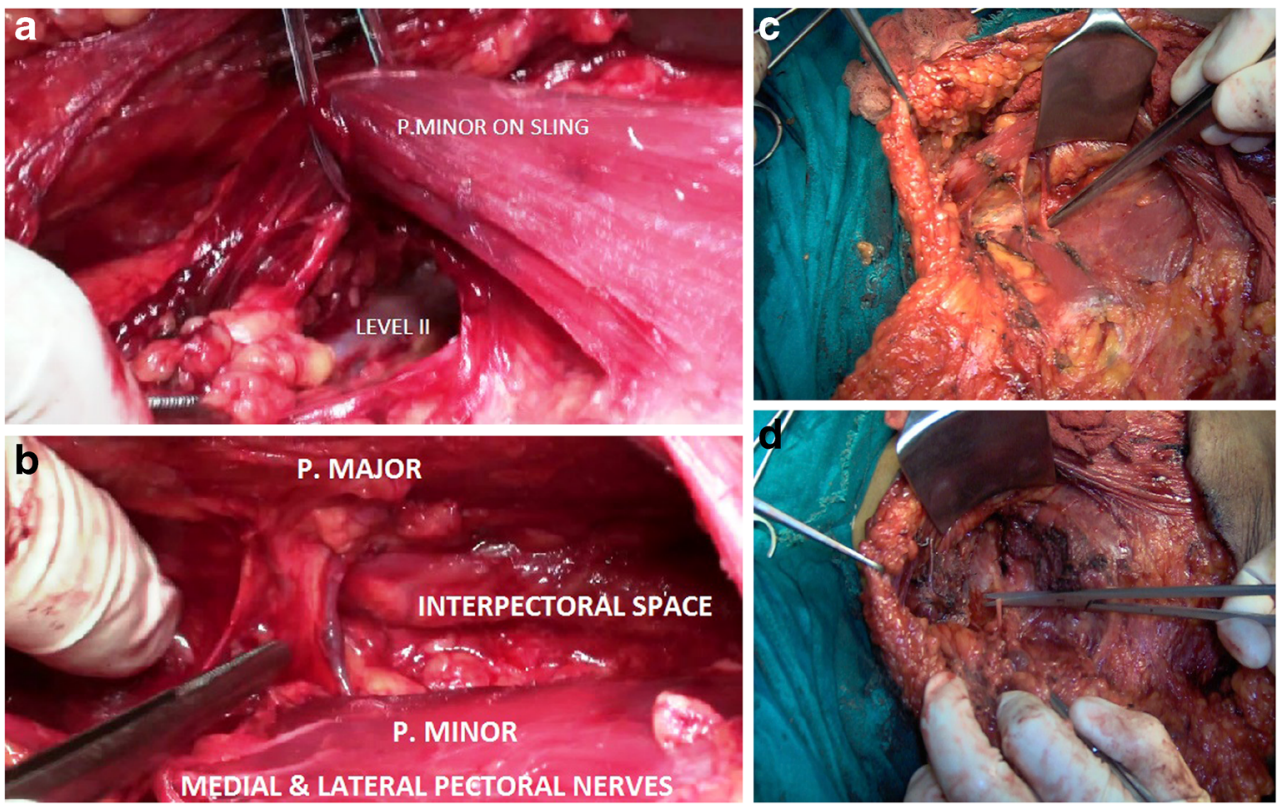


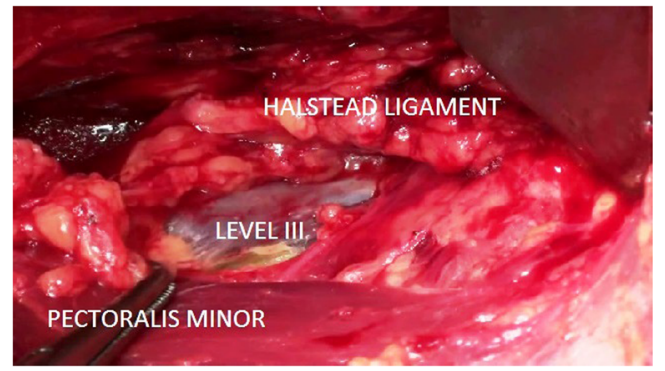

Fig. 6 The retraction of the pectoralis minor muscle facilitates the dissection in the region of level-III. The medial limit of axillary dissection, the "Halstead's Ligament" can be seen. The entire fibro-lymphatic tissue right up to the axillary vein needs to be dissected exposing but not denuding the axillary vein of its sheath

denude the vein of its sheath completely as this may be associated with a higher incidence of axillary vein thrombosis. The lateral anatomical limit of level-II dissection is the lateral thoracic vessels, which may be preserved or ligated and transected (Fig. 7). The dissection of the fibro-lymphatic tissue at level-I proceeds laterally right up to the thoracodorsal pedicle and the latissimus dorsi (LD) tendon (both these structures form the lateral anatomical limit of level-I axillary dissection and must be preserved) (Figs. 8 and 9).

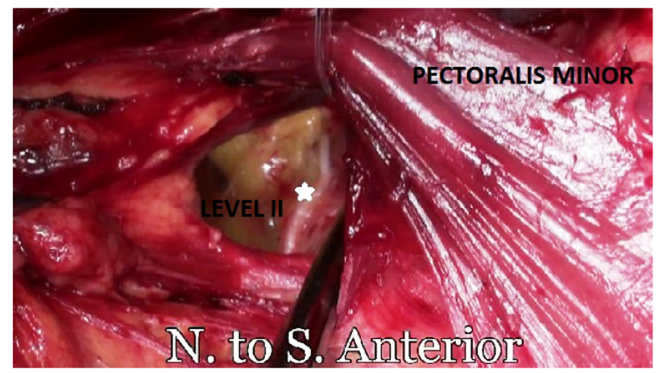

Fig. 7 Level-II dissection in progress. The pectoralis minor muscle is now retracted medially to expose leve-II. The lateral limit of this dissection is up to lateral thoracic vessels. The nerve to serratus anterior appears as the dissection proceeds laterally

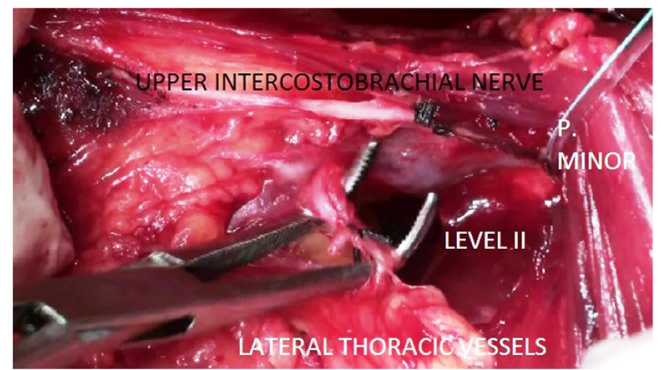

Fig. 8 The lateral limit of level-II dissection, the lateral thoracic vessels and also the upper most intercostobrachial nerve are seen. The lateral thoracic vessels may need to be dissected off all the fibro-lymphatic tissue and may occasionally need to be transected as is shown
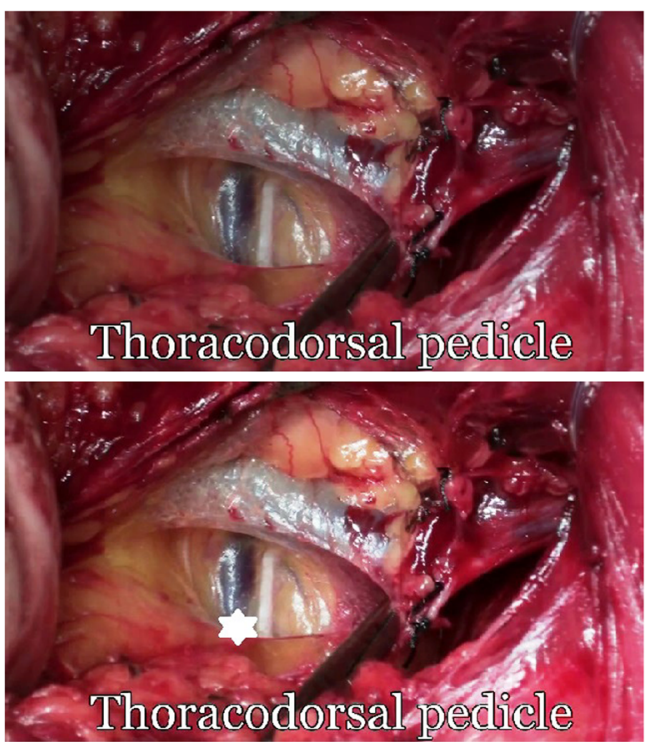

Fig. 9 a As the dissection proceeds laterally the thoracodorsal pedicle (the subscapular vein and thoracodorsal artery and nerve) becomes visible in the depth. b The axillary vein, the superior most limit of axillary dissection may also be seen

\section{Commandment-7-Intercosto-Brachial Nerves (IBN)}

These nerves are three or sometimes more than three in numbers and should to be preserved if not directly involved by cancer (nerve preserving ALND). It is also recommended to stay below the superior most IBN in order not to interfere with the lymphatic drainage of the upper extremity. Keeping dissection below the superior most IBN has been observed in various studies to be associated with lower incidence of upper limb lymphedema [4-6]. If one decides to sacrifice the intercostobrachial nerves, it is recommended that a cold knife rather than diathermy should be used to prevent postoperative neuralgia (Fig. 10). Sacrificing this nerve leads to an annoying hypoesthesia on the medial aspect of the upper arm. It is also mandatory to identify and preserve the nerve to serratus anterior before sacrificing the IBNs (Fig. 10). Nerve to serratus anterior lies along the chest wall and shows up automatically while dissecting in a medial to lateral direction below the axillary vein. Tracing the tendon of latissimus dorsi (LD) up to the point where it crosses the axillary vein is also a landmark to find this nerve. Some surgeons like to trace back the pectoral pedicle up towards the axillary vein to find this nerve (Fig. 5b).

Angular vein is a reliable spatial landmark of the lower limit of axillary dissection. The author has used this landmark in a large number of cases and found it to be constant and reliable in location [5]. 


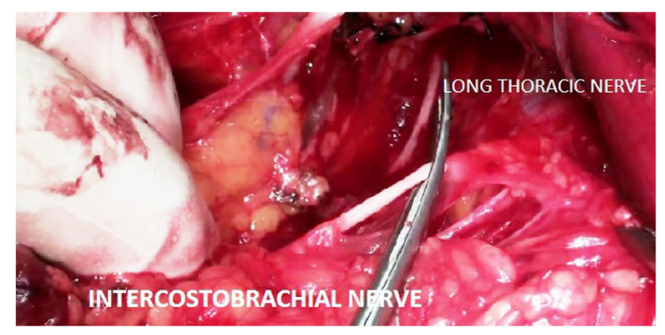

Fig. 10 If the intercostobrachial nerves have to be sacrificed due to their infiltration by the disease, it is advisable to use cold scissor rather than diathermy to prevent the formation of neuromas leading to postoperative neuralgias. It is also mandatory to demonstrate the nerve to serratus anterior (SA) before transecting the nerve. The nerve to SA can be seen

\section{Commandment-8 - Reaching the Lateral and Lower Limit of Axillary Dissection}

Dissection of level-I involves removal of all fibro-lymphatic tissue between the lateral thoracic vessels (or lateral border of pectoralis minor) to the thoracodorsal pedicle and tendon of LD muscle. The author likes to lift this pedicle on a vascular sling or a strip of disposable surgical glove to preserve it and to prevent neuropraxic injury to the nerve to LD while dissecting around it (Fig. 11). This maneuver also facilitates an optimal clearance right down to the lower limit of axillary dissection, i.e., the Angular vein which drains the chest wall and joins thoracodorsal vein to form the subscapular vein (Fig. 12).

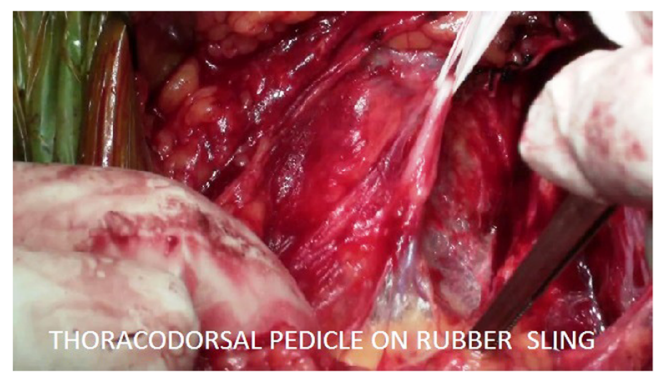

Fig. 11 The thoracodorsal (TD) pedicle is now lifted on a rubber sling in order to facilitate the dissection of all lymphatic tissue around this pedicle down to the lower limit of axillary dissection. It takes by some authors as the lateral limit of ALND; however, often there are nodes lateral to this pedicle, it is safer to dissect laterally up to the $\mathrm{LD}$ tendon

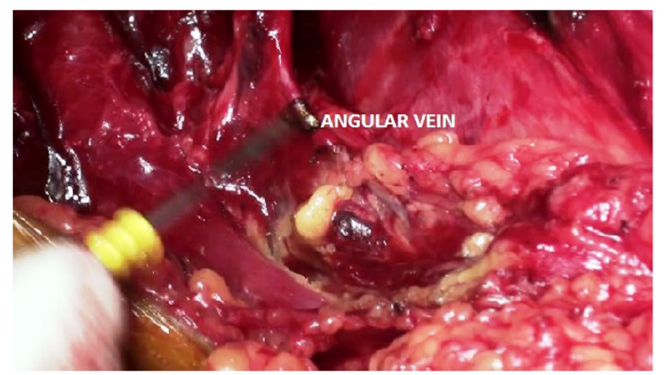

Fig. 12 Angular vein, arising from the chest wall and joining the thoracodorsal vein to form the subscapular vein can be seen. This vein is reliable landmark for the lower limit of ALND
(Some surgeons like to take the point of entry of thoracodorsal nerve into the LD muscle as lower limit of axillary dissection).

Preservation of thoracodorsal pedicle is very important if LD flap has to be used for immediate breast reconstruction.

\section{Commandment-9-Achieving and Demonstrating all Anatomical end Points of ALND (Fig. 13)}

It is mandatory to achieve, revisit, and demonstrate the anatomical end points of axillary dissection to the surgical assistants as a part of routine surgical protocol. The medial limit of axillary dissection is the Halstead's ligament, upper limit being the axillary vein, the thoracodorsal pedicle is the lateral limit and angular vein may be considered the lower limit [5].

\section{Commandment-10 - Closure of the Incision with Half Suction Drain(s) in situ (Fig. 14)}

After a thorough saline wash, the wound is closed after leaving two suction drains (or only one) in situ, one each for the axilla and flaps. The author in a randomized controlled study (ISRCT $\mathrm{N}$ number-24484328) observed that the seroma rates are lower with the use of half suction rather than full suction drains [6].

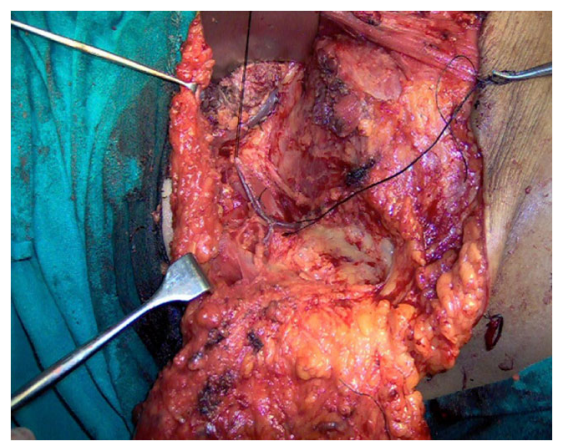

Fig. 13 The complete extent of ALND can be seen. The lateral limit being the TD pedicle, lower limit, the angular vein, the superior limit being the AV and the medial limit is considered to be the Halstead's ligament

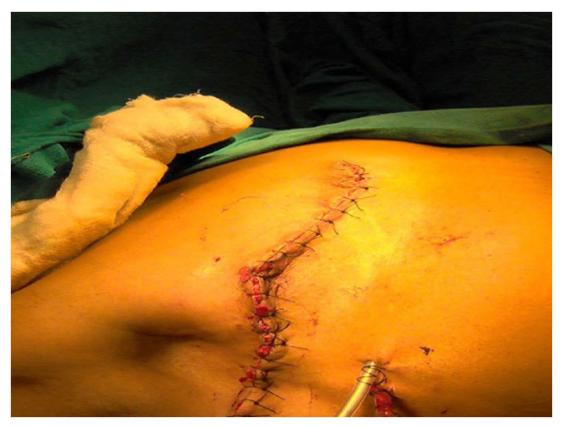

Fig. 14 The incision is closed with suction drains in situ 
After applying the usual dressings, some surgeons like to apply axillary padding and arm strapping to reduce the seroma formation; however, there is no consensus on any of these maneuvers. The author recommends encouraging the patient to start with shoulder and arm exercises as soon as possible. This, combined with the use of half suction drainage has been found to significantly reduce seroma formation in a randomized controlled study conducted by the author [6]. The use of diathermy as opposed to cold knife has also not been found to make any significant difference in seroma rates [6].

\section{References}

1. Chintamani (2013) The paradigm shifts in the management of breast cancer-Have we finally arrived? Indian J Surg 75(6):419-423. doi: $10.1007 / \mathrm{s} 12262-013-1022-1$
2. Chintamani, Singh JP, Mittal MK, Saxena S, Bansal A, Bhatia A, Kulshreshtha P (2005) Role of p-glycoprotein expression in predicting response to neo-adjuvant chemotherapy in breast cancer-a prospective clinical study. World J Surg Oncol 3:61

3. Chintamani, Jha BP, Anju B, Saxena S, Bhatnagar D (2007) The expression of mismatched repair genes and their correlation with clinicopathological parameters and response to neoadjuvant chemotherapy in breast cancer. Int Semin Surg Oncol $4: 5$

4. Chintamani, Singhal V, Singh JP, Lyall A, Saxena S, Bansal A (2004) Is drug-induced toxicity a good predictor of response to neo-adjuvant chemotherapy in patients with breast cancer? a prospective clinical study. BMC Cancer 4:48

5. Chintamani (2010) Optimum Axillary Dissection in Breast Cancer-is Angular Vein a Reliable Spatial Landmark? Indian J Surg Oncol 1(3): 214-215, 10.1007

6. Chintamani (2005) Half versus full vacuum suction drainage after modified radical mastectomy for breast cancer-a prospective randomized clinical trial -ISRCTN24484328. BMC Cancer $5(1): 11$ 